\title{
Representaciones sociales de lectores opinantes en prensa digital sobre el caso Marina Kué (2012-2014)
}

\section{Social representations of readers in digital press on the case Marina Kué (2012-2014)}

\author{
Karina Elizabeth Báez Houdin ${ }^{a}$
}

\begin{abstract}
Resumen
El Caso Marina Kue puede ser considerado uno de los problemas sociales más graves en la última década en Paraguay, vivido por el campesinado, donde fallecieron 11 campesinos y 6 policías, tras un dudoso operativo fiscal-policial en el año 2012, con una alta repercusión política a nivel nacional e internacional. La presente investigación tuvo como objetivo analizar las representaciones sociales expuestas por lectores/as de medios a través de sus comentarios en la WEB de dos medios de prensa de mayor difusión en el periodo 2012-2014. La metodología planteada fue exploratoria, de enfoque cualitativo. El estudio incluyó 5.734 comentarios de lectores opinantes en 331 artículos correspondientes a cinco momentos claves del proceso. Los datos fueron analizados a través de técnicas de análisis de contenido, con procedimientos de codificación y categorización en base a sustentos teóricos previos. Como resultado se encontró que la mayoría responsabilizó al gobierno del ex presidente Fernando Lugo de lo ocurrido entre campesinos y policías. Así también, afirman que si bien existían críticas y decepción hacia el gobierno, el Congreso Nacional procedió de manera irregular a destituir al presidente a través de un juicio político realizado en tiempo récord sin darle tiempo para la preparación de su defensa. Descalificaron la gestión del Parlamento y de los partidos políticos al no promover acciones que lleven adelante un buen sistema de gobierno y de involucrarse directamente en gestar un golpe de estado disfrazado, con legalidad pero sin legitimidad. Sobre la categoría de Justicia, ésta se expresó como práctica invisible en la problemática cuestionando su no proceder de manera rápida para detener a los campesinos. En la temática de la "distribución de la tierra", ubicaron a los campesinos como criminales, haraganes e invasores. En la categoría de "actores involucrados", se centraron en los problemas entre campesinos y policías, campesinos y propietarios, colocando a campesinos como únicos responsables por no respetar la propiedad privada. La categoría "escenarios" durante el proceso del Caso Marina Kue, según las representaciones de lectores/as fueron
\end{abstract}

Kera Yvoty: reflexiones sobre la cuestión social. Vol. 1, 2016, 118-126.

ISSN (impreso): 2519-7797

a Universidad Nacional de Asunción, Instituto de Trabajo Social, Paraguay.

Correspondencia a: kari.houdin@gmail.com

Cita:

Báez Houdin, K. E. (2016). Representaciones sociales de lectores opinantes en prensa digital sobre el caso Marina Kué (2012-2014). Kera Yvoty: reflexiones sobre la cuestión social, 1, 118-126.

Recibido: 20 julio 2016

Aceptado:

22 setiembre 2016 
espacios democráticos donde se permitió la utilización del juicio político y se encontraron distintas expresiones de descontento a las representaciones políticas partidarias. El "problema de la tierra" podría ser solucionado a través de un programa de reforma agraria aunque se hizo mayor énfasis en el respeto a la propiedad privada. En lo que respecta a los "intereses y disputas" se visualizó un alto grado de descalificación, insultos y críticas hacia las publicaciones y el propio medio y en menor medida, a otros/ as lectores/as. En base a comentarios en las publicaciones, surgieron nuevas ideas y pensamientos acerca de lo ocurrido como que esto haya sido un desvío de atención cuando en el país se estaba tratando temas importantes como el desbloqueo de la llamada "lista sábana", que produjo varias movilizaciones al momento de los sucesos.

Palabras clave: caso Marina Kué, representaciones sociales, lectores opinantes.

\begin{abstract}
Abstrac
The Marina Kue case can be considered one of the most serious social problems experienced by the peasantry in the last decade in Paraguay, where 11 peasants and 6 policemen died after a dubious fiscal-police operation in 2012, with a high political impact national and internationally. This research aimed to analyze the social representations exposed by newspapers readers through their comments on the website of two newspapers with the largest circulation in the period 2012-2014. The methodology was exploratory with a qualitative approach. The study included 5,734 comments from opinion readers in 331 articles corresponding to five key moments of the process. The data were analyzed through content analysis techniques, with coding and categorization procedures based on previous theoretical support. As a result, it was found that the majority blamed the government of former president Fernando Lugo for what happened between the peasants and the police. Likewise, they affirmed that although there were criticisms and disappointment towards the government, the National Congress proceeded in an irregular way to dismiss the president through an impeachment carried out in a record time without giving him time to prepare his defense. The readers disqualified the management of the Parliament and political parties by not promoting actions that carry out a good governmental system and directly engaging in a disguised coup d'état, with legality but without legitimacy. On the category of Justice, it was seen as an invisible practice in the problem questioning it for not proceeding quickly to stop the peasants. In the theme "distribution of land", they placed peasants as criminals, lazy and invaders. In the category of "actors involved", they focused on the problems between the peasants and police, peasants and landowners, placing peasants as solely responsible for not respecting private property. The category "scenarios" during the Marina Kue Case process, according to readers' representations, were democratic spaces where the use of the impeachment was allowed, and different expressions of discontent were found towards party political representations. The "land problem" could be solved through an agrarian reform program, although more emphasis was placed on the respect for private property. In relation to "interests and disputes" a high degree of disqualification, insults and criticism towards the publications and the newspaper itself and, to a lesser extent, towards other readers were visualized. Based on the comments of the publications, new ideas and thoughts arose about what happened like that it was a diversion when the country was dealing with important issues such as the unblocking of the so-called "sheet list" which produced several demonstrations at the time of the events.
\end{abstract}

Keywords: Marina due case, social representations, newspapers readers. 


\section{Introducción}

El desarrollo de la comunicación se ve potenciado gracias a los avances tecnológicos, sobre todo en internet donde se instalan espacios virtuales en el que las personas comparten contenido, ideas, opinioznes sobre diversos temas ya sean culturales, sociales, económicos y políticos. Es en este espacio, donde se construyen y afianzan las representaciones sociales, visualizando éstas en términos de Castells (2009) como históricas y novedosas ya que al articularlas en un texto digital y al ser interactivo el espacio, se integran, mezclan las expresiones culturales, a través de uno de los elementos más influyentes y de mayor propagación en un sistema social, el pensamiento humano (Benkler, 2006 citado en Castells, 2009, p.50).

Los medios de comunicación funcionan como uno de los principales canales de información y a su vez cumplen un rol de mediación entre las representaciones sociales que el medio presenta sobre ciertos fenómenos sociales a través de editoriales, columnas de opinión, artículos para dar a conocer situaciones, hechos, acontecimientos y la participación de lectores que a su vez generan sus propias representaciones sociales. En ese sentido, las noticias emitidas por los medios de prensa sobre este problema social en todo el proceso de su ocurrencia, juicio, protesta social, ha sido no sólo abundante sino además generó interacciones importantes entre las y los lectores opinantes y la sociedad en general.

El trabajo aborda los conceptos del teórico Moscovici (1979) quien sostiene que las representaciones sociales prestan mayor atención al pensamiento común, conocimientos y creencias para comprender un objeto en determinado grupo social a través de un proceso de comunicación.

$$
\text { Esta investigación pretende }
$$
visualizar las representaciones sociales expuestas por lectores opinantes, a través de sus comentarios en las plataformas digitales de los diarios ABC Color y Última Hora considerando como tema central de estudio el Caso Marina Kue, que puede ser considerado como uno de los problemas sociales más graves en la última década del país, vivido por el campesinado paraguayo, donde fallecieron 11 campesinos y 6 policías tras un dudoso operativo fiscal-policial en el año 2012 con una repercusión muy alta a nivel político. Este acontecimiento marcó la finalización en tiempo récord por medio de un juicio político a un gobierno que aglutinaba a partidos y movimientos sociales tanto de derecha como izquierda electo por la mayoría a través del voto en elecciones generales tras 60 años de gobierno del Partido Colorado.

\section{Objetivo general}

Analizar las representaciones sociales expuestas por los/las lectores/as a través de sus comentarios en la WEB 2.o de los diarios ABC Color y Última Hora sobre el caso Marina Kue - Curuguaty en el periodo 2012-2014.

\subsection{Objetivos específicos}

-Categorizar las representaciones sociales expuestas por lectores/as a través de sus comentarios en los espacios que otorgan los artículos publicados.

-Identificar similitudes y diferencias de las representaciones sociales construidas por lectores a partir de sus comentarios en cada uno de los medios de prensa seleccionados. -Ubicar cuál de los dos diarios estudiados presenta una mayor interacción entre lectores/as.

\section{Materiales y métodos}

Tipo de estudio: Exploratorio, descriptivo de carácter cualitativo.

Población y Muestra: Por conveniencia. A raíz del gran volumen de datos hallados (18.582 opiniones de lectores halladas en 331 artículos) se optó por establecer tres criterios de inclusión y exclusión: a) Siete periodos claves del Proceso Caso Marina Kue durante los 
años 2012-2014; b) Comentarios del día del hecho, acontecimiento relevante y dos días consecutivos; c) Comentarios primarios de cada artículo. Con esto, se redujo la cantidad de comentarios a 5.734 . Para el análisis final, se utilizaron sólo 904 comentarios. La exclusión de los más de 4000 comentarios se debió a que éstos presentaban agresiones, insultos o bien, eran ideas muy descontextualizadas, propagandas, alusiones a vídeos $\mathrm{u}$ otros artículos, llamados a movilizaciones, etc. Los comentarios que refieren a los periodos establecidos pudieron ser extraídos en su totalidad del portal digital del Diario ABC Color, no así en el portal del diario Ultima Hora, debido a que la Web de este periódico sufrió una renovación de plataforma en julio del 2013 en la que solo realizaron la migración de las publicaciones del medio, no así de los comentarios de lectores por una cuestión técnica. Los periodos seleccionados fueron establecidos a partir de un pre análisis realizado a documentaciones y publicaciones de medios referentes al Caso Marina Kue.

Periodos claves para la definición de la muestra: a) Acontecimiento del hecho (15 al 17 de junio de 2012); b) Juicio político al Presidente Fernando Lugo (22 al 24 junio 2012); c) Acusación de la fiscalía (16 al 18 de diciembre 2012);. d) $1^{\text {a }}$ Audiencia preliminar (15 al. e) Cinco campesinos presos inician su segunda huelga de hambre (14 al 16 febrero 2014); f) Intervención de la iglesia (8 al 10 marzo 2014), g) INDERT reconoce que las tierras de Marina Kue son del Estado Paraguayo (15 al 17 mayo 2014).

Recolección de los datos: La técnica utilizada para la recolección de información fue la de análisis de contenido cualitativo. Se estableció una matriz inicial de recolección, en la que se fijaron los primeros insumos para el pre-análisis; una ficha electrónica, donde se muestra el artículo seleccionado para el análisis de contenido con sus respectivos comentarios. Cada comentario, cuenta con una codificación a fin de conocer su procedencia, es decir, a qué artículo, periodo de análisis y medio de prensa corresponde. Categorías de Análisis: A partir de los antecedentes recogidos en el Marco Teórico, se establecieron algunas dimensiones y categorías que por su relación con el Caso Marina Kue se consideraron como de gran importancia, posteriormente fueron agregándose otras durante el proceso de recolección a fin de visualizar todas las ideas expuestas en el análisis de la información extraída de los comentarios de lectores.

Dimensiones y categorías: Poderes del Estado (Ejecutivo, Legislativo, Judicial); Actores Involucrados (Campesinos sin tierra, Policías, Propietarios, Fiscales, Organizaciones sociales, Empresas); Sistemas de gobierno (Democracia, Dictadura); Propiedad de la Tierra (Privada, Pública); Intereses o Disputas (Acuerdo basado en un argumento $\mathrm{u}$ opinión, Insultos. Descalificaciones. Desacuerdos, Interpelación entre lectores/as, Adición de otros puntos de vista).

\section{Resultados}

\subsection{Representaciones sociales en torno a los Poderes del Estado}

En esta dimensión se analizaron 300 comentarios, el 66\% (198 comentarios) responsabiliza al gobierno de Lugo como el principal responsable de la muerte de 17 personas en Marina Kue, así como también de ser cómplice de las ocupaciones de tierra por parte de campesinos; incluyendo un nuevo elemento en la discusión; la criminalización del campesinado a quienes consideran como "omnipotentes" y que contaban con la venia del Ejecutivo para invadir propiedades, exigir subsidios, etc.

$$
\text { El 22\% (66 comentarios) ubica }
$$
al Legislativo como responsables desde distintas matices pero señalando su injerencia en ella. En este ítem, la mayoría de los comentarios mostraba una indignación ante el cambio tras 60 años de gobierno del Partido Colorado sobre todo considerando que en todo ese tiempo, no se había generado una matanza de tal magnitud 


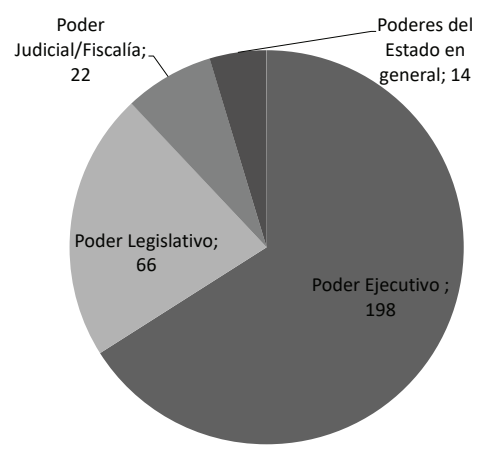

Figura 1: Ideas sobre los Poderes del Estado

entre policías y campesinos. En cuanto al Poder Judicial, los comentarios indican que este poder está prácticamente invisibilizado tanto en los artículos seleccionados como en los propios cometarios. Tan sólo el $7 \%$ (22 comentarios) hace relación a alguna vinculación o responsabilidad de la Justicia en el Caso Marina Kue. La responsabilidad de la Justicia gira en tornoa la incapacidad de condenar a los así como también, cómplice de la destitución de Fernando Lugo del poder al haber aprobado una supuesta orden de desalojo de una propiedad considerada del Estado Paraguayo. Las ideas vinculadas a la responsabilidad del Estado se encuentra un 5\% (14 comentarios) donde los lectores en su mayoría ubican al Estado como un ente desarticulado en el cual uno de los poderes -Ejecutivo- debe responder y actuar para solucionar un problema que indudablemente necesita la intervención de todos los poderes. Los demás poderes del Estado, son cuestionados por situaciones efímeras que no se vinculan directamente con lo ocurrido en Marina Kue.

\subsection{Representaciones sociales en torno a actores involucrados}

Según las opiniones de lectores/as, los principales actores dentro del proceso Marina Kue son los campesinos, policías, propietarios obviando a la Fiscalía y sector empresarial, quienes llamativamente no han tenido relevancia. Se tuvo en cuenta 327 comentarios relacionados a esta dimensión en la que se expone en primer
"Liiindo el "Cambio" que trajo Lugo y la zurda caviar! Que le culpen a los gobiernos anteriores es un chiste! Nunca hubo una matanzaasi en ninguno de esos gobiernos por enfrentamientos con campesinos!". $C_{3} A_{51} P_{1} A B C$

"La verdad quien está fallando demasiado como siempre es LA JUSTICIA.estos están libres por eso los ministros de la Corte son los responsables directos de dejar que esta gente esté libre no me digan $q$ un fiscal no tiene potestad de actuar con esta gente". $C_{20} A_{11} P_{1} A B C$

lugar la participación de los campesinos desde dos ideas centrales, la primera desde la responsabilidad ante lo ocurrido y la segunda desde colocarlos como víctimas de lo ocurrido, esto corresponde a un 38\% (124 comentarios) del total de comentarios donde la mayoría de estos criminaliza a la lucha campesina por la tierra además de culpabilizar a los campesinos de iniciar la balacera en Marina Kue.

En segundo lugar, existe un $27 \%$ (89 comentarios) que vinculan directamente en lo ocurrido a los propietarios de tierras, en este caso al ex dirigente colorado y empresario en la matanza, a través de terceras personas ya sea intervención de grupos paramilitares o francotiradores. La percepción en relación a la participación de policías, ubicada en tercer lugar con un $26 \%$ (85 comentarios) da cuenta de una división entre quienes piensan que son responsables de lo ocurrido por un error en el procedimiento y quienes creen que son víctimas de lo ocurrido, esta última versión es la predominante. Por último, encontramos en cuarto lugar que el 6\% (19 comentarios) menciona a organizaciones sociales y en quinto lugar un $3 \%$ (10 comentarios) a la intervención de fiscales.

\subsection{Representaciones sociales sobre Sistemas de Gobierno}

La dimensión sistemas de gobierno englobaadoscategorías desuma importancia para nuestro análisis, la primera hace referencia al sistema de gobierno dictatorial 


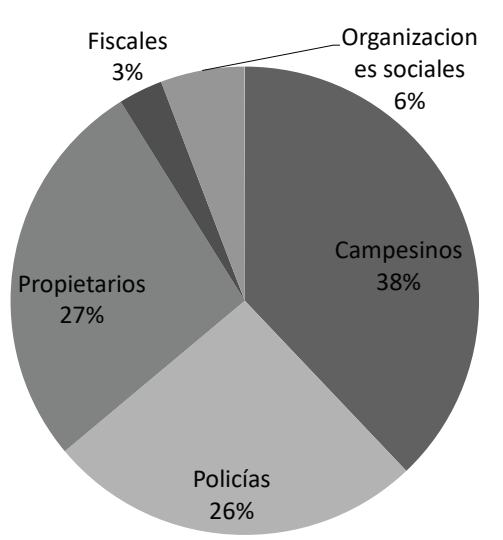

Figura 2. Ideas claves sobre actores involucrados

llevado a cabo durante 35 años entre los años 1954 - 1989 bajo el mando de Alfredo Stroessner, dejando como saldo más de 2000 desaparecidos quienes habían sido tildados de opositores y comunistas, colocando como categorías de análisis la reivindicación a la dictadura y la oposición a esta. Por otro lado, se encuentra la apertura democrática, iniciado a partir del derrocamiento del gobierno dictatorial liderado por Andrés Rodríguez, donde se fue instalando un proceso de participación política en nuestro país. Ahora bien, en este contexto es donde se permite establecer distintos escenarios sin que ello signifique un quiebre democrático, es decir, a poder analizar el caso Marina desde lo acontecido en materia política y social tomando a consideración el rol protagónico de los partidos políticos y sus representantes en el Congreso Nacional.

Enun 97\%(109comentarios) sedebate en torno a los escenarios que se generaron tras lo acontecido el 12 de junio de 2012 en Marina Kue en un contexto democrático donde se dan varias tendencias, una de ellas tiene que ver con las falencias de los partidos políticos, se menciona también que hubo una alianza de partidos políticos para poder realizar "un golpe de Estado disfrazado" donde la mayoría asegura estar en desacuerdo con el gobierno de Lugo y los partidos políticos que lo apoyan, sin embargo, señalan que existió un montaje
"No todos los policías son malos y a estos carperos no se les puede llamar pobre, el pobre es aquel que sale a buscar trabajo para darle de comer a su flia , estos son araganes y delinquentes, les guste o no necesitamos otro como Stroessner para cortar de raíz a estos bandidos asesinos". C44A118ABC

"NN..estos campesinos son sinvergüenzas. no les importa nada y luego se hacen los inocentes, son muy agresivos y van a matar a cualquiera para lograr sus objetivos y cuando consiguen las tierras las venden nuevamente.. no defiendan a esta gente que quiere que se le regale todo, que trabajen si quieren tener algo haraganes cuanta gente humilde hay, que dia a dia se sacrifica para salir adelante, y esta gente quieren conseguir a la fuerza..yo no voy a salir en defensade unos crriminales haraganes". $C_{14} A_{2} P_{1} A B$

o conspiración para tomar el poder por la fuerza. Así también, que las medidas adoptadas por el Congreso Nacional corresponden a un hecho inconstitucional, pues no otorgaron el tiempo suficiente al Presidente en ejercer su defensa. Tan sólo el 3\% de los comentarios hace relación a alguna reivindicación a la dictadura de Stroessner a fin de solucionar los conflictos que se habían suscitado.

\subsection{Representaciones sociales sobre propiedad de la tierra}

El trasfondo del episodio ocurrido en Marina Kue, tiene relación directa con la situación más estructural de la distribución de la tierra en nuestro país, debido a que ningún gobierno después de la dictadura pudo encarar el problema con visos de solución, sino que ha empeorado a través de la aprobación de políticas públicas por parte del Congreso Nacional que hasta el momento han servido sólo para la expansión de la frontera agrícola y la profundización del modelo de commodities.

Se ha encontrado un total de 44 comentarios en los que se debate la situación actual del país en cuanto a la distribución de la tierra. En relación a las tierras públicas, $37 \%$ (16 comentarios) aseguran que el problema central está en tres aspectos claves, la falta de una reforma agraria integral, una renovación de los 
"digan de nuevo de burla! "ÉRAMOS FELICES Y NO LO SABIAMOS"y?? clarisimo que eramos felices. hubo errores en la época stronista si sr como hay ahora miles dee atrocidades.pero POPR QUE SUPONEN QUE STROESSNER JAMAS PERMITIO QUE se agrupen estos "Campesinitos pynandy" pobrecitos angá?' por que creen que jamas permitio las ligas agrarias". C48A125 $P_{2} A B C$

"pobrecitos estos zurditos de mierda pensaron que eran dueños del país y se ivan a quedar 60 años en el poder, jajajaja ni 4 años completaron. Eso les da pauta que el Paraguay es para que lo gobiernen verdaderos machos(...)". C $7 A_{12}{ }_{5} P_{2} A B C$

registros catastrales y la recuperación de tierras públicas en manos de quienes no son sujetos de la reforma agraria. Se da una crítica hacia el gobierno de Lugo, ya que según algunos lectores, el ex presidente había utilizado como base de campaña a la reforma agraria integral. Así también, se debate sobre la propiedad privada en un $64 \%$ ( 28 comentarios) y en ese sentido, al otorgamiento de las tierras a través del mecanismo de usucapión, donde muchos comentarios indican que fueron los políticos y empresarios quienes, a través de sus conexiones con la justicia son adjudicados con tierras pertenecientes al Estado. En cuanto a la propiedad de Marina Kue, los lectores adjudican la propiedad de la tierra a Blas N. Riquelme y que ante este hecho se debe respetar la tenencia evitando un conflicto social.

\subsection{Representaciones sociales en torno a intereses o disputas}

En cuanto a los intereses y disputas, se tiene en cuenta que en la cuestión de fondo siempre está en el poder, puesto que la sociedad está compuesta por sectores y organizaciones que conviven entre sí y donde tienen intereses contrapuestos.

En esta dimensión, se visualiza algunas reacciones de lectores en torno a las publicaciones realizadas por los medios de prensa seleccionados donde se

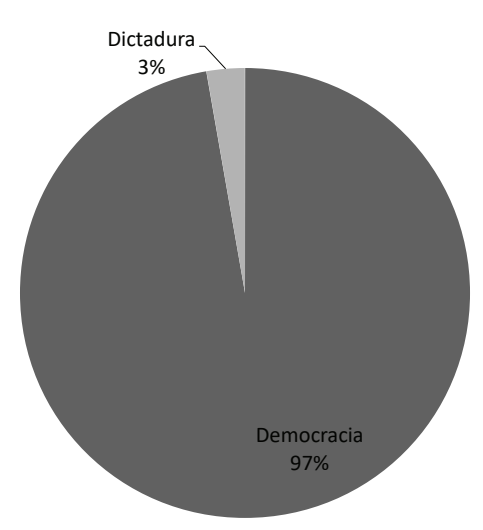

Figura 3. Ideas claves sobre Sistemas de Gobierno

consideran cuatro elementos de análisis, en primer lugar se encuentran los comentarios que tienen connotación peyorativa $y$ descalificativa hacia la opinión de otras personas, las publicaciones del medio y quien lo dirige. Esto corresponde a un $63 \%$ (7o comentarios) donde la mayor crítica de lectores se da en relación a las publicaciones, al medio de prensa en sí y hacia la figura de su director, en su mayoría, presentaban desacuerdos al Diario ABC Color no así al Diario Última Hora en 66 comentarios y un pequeño número de 4 comentarios hace una alusión a insultos entre lectores.

\section{Discusión}

Considerando las representaciones halladas es posible pensar que la realidad social expuesta en los artículos es presentada desde una perspectiva segmentada que posibilita a los lectores a construir y plantear opiniones desde la perspectiva del medio, incluso tomando casi como única referencia los artículos publicados, ubicándolos aparentemente como fuentes primarias del acontecimiento y por lo tanto centran el debate, la crítica y la opinión en el contenido producido por los medios dejando de lado todo el contexto social de lo ocurrido. Aparentemente, la simplificación de un problema estructural como lo es la distribución de la tierra en nuestro país, en este caso aparece como 


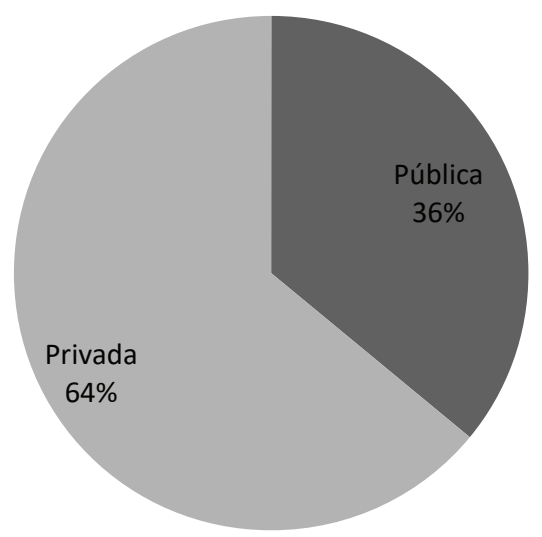

Figura 4. Ideas sobre propiedad de la tierra

"Marina Cué es del estado, y es tierra para la reforma agraria. En huelga las víctimas. Y los verdaderos responsables de la masacre, seguramente, en libertad, en la oscuridad y en impunidad. 17 compatriotas allí fueron acribillados. Esta masacre ha sido utilizada para tumbar la democracia en el Paraguay". $C_{4} A_{1} P_{5} A B C$

"Esta gente murio al pppp...al final se dice ahora lo Ellos reclamaban justamente por ser moradores de la zona.. Hay que hacer justicia ya”. $C_{2} A_{1} P_{7} A B C$

una situación o problema entre dos actores (campesinos y propietarios) mediados por un tercero (la policía) por lo menos desde las publicaciones halladas en los medios de prensa analizados.

La funcionalidad de las representaciones sociales permite una articulación de los saberes comunes entre los sujetos dentro de un proceso de construcción de una realidad que puede o no ser compartida por la mayoría de los mismos. Esta situación favorece a la regulación y equilibrio en donde se da un mutuo reconocimiento y pertenencia de sus comportamientos en un espacio social. Se puede entrever en el contexto de este estudio que existe un carácter político - organizacional manifestado a través de las representaciones sociales en tanto se den procesos de participación y diálogos basados en la reflexión y crítica que configuran un panorama propio de una sociedad democrática. De alguna manera, los medios de comunicación se presentan a sí mismos como transmisores objetivos de la realidad social centrando su rol en la distribución delainformacióna la población en general. Sin embargo, es bien sabido que existe todo un sistema de producción de noticias donde éstas son transmitidas según una selección de ideas, segmentación y combinación de representaciones sociales sobre un hecho oacontecimiento específico. Es por ello que existe un alto porcentaje de representaciones sociales negativas tanto hacia las publicaciones de los medios como a quienes lo dirigen.

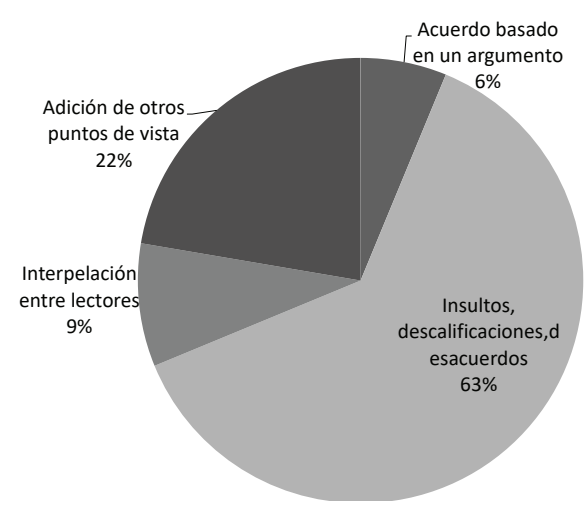

Figura 5. Ideas sobre intereses y disputas

“GENTE DESVIO DE ATENCION.. ESO SE LLAMA ESTOOOOO.PORQUE? POR ESTOS TEMAS..1 LISTAS SABANA. 2 DESVIO DE Cajubi asi nomas.. MIREN TODOS LOS PERIODICOS EN TODAS PARTES HABLAN DE lo de CURUGUATY que es triste.. pero.. eso fue todo PLANEADO.... ES CUESTION DE PENSAR..... C $C_{7} A_{75} P_{1} A B C$

"La información es pura anécdota..es lo que uno puede Comentar entre amigos en una ronda de terere..y se publica aquí como la GRAN NOTICIA. Disculpen eso es mediocridad profesional(...). C17A186ABC

\section{Conclusiones}

Se reconoce al entorno virtual como un espacio de discusión frente a la opinión pública, a contextos sociales, políticos y culturales entre otros ámbitos en donde se interactúa y se informa de temas de interés general con la posibilidad de conocer y discutir la realidad del país. En ese sentido, los diarios ABC Color y Última Hora en sus formatos Web 2.0 cuentan 
con espacios que abren la posibilidad a los lectores de interactuar libremente, sobre todo con el acceso a redes sociales. Así mismo, mucha información que maneja la sociedad paraguaya sobre la realidad del país proviene de los medios de prensa, los cuales difunden diversas representaciones sociales. Por esta razón puede afirmarse que con lo que leen en las publicaciones afianzan sus tendencias ideológicas, las manifiestan y consolidan cada vez que interactúan publicando sus comentarios.

El portal del diario $A B C$ Color fue el medio que mayor destaque le dio a todo lo relacionado con el Caso Marina Kue publicando 314 artículos con un total de 18.421 comentarios entre primarios y secundarios. Por su parte el diario Última Hora publicó un total de 20 artículos en los que se encontraron 181 comentarios entre primarios y secundarios, considerando todos los comentarios que pudieron haberse dado entre el 12 de junio de 2012 y el 13 de julio de 2013. Al hablar de similitudes y diferencias entre comentarios de lectores en los dos medios de prensa analizados, se visualizó que tanto en la plataforma de $\mathrm{ABC}$ Color como en la de Última hora se dio un amplio debate en torno a los 7 periodos tomados en cuenta para la recolección de datos, habiendo mayor cantidad de comentarios en el Diario ABC Color. En este último, se observa que la mayoría de los lectores opinantes expresan representaciones sociales negativas tanto hacia el ex presidente Lugo, a los campesinos, y los partidos de izquierda, utilizando en su mayoría un lenguaje vulgar y soez.

En el Diario Última Hora, analizado a partir del cuarto periodo, se da como un cuestionamiento mayor hacia la actuación de la fiscalía y los jueces que llevan adelante el Caso Marina Kue, además de cuestionar la falta de pruebas para la acusación de campesinos en los asesinatos desarrollados el 12 de junio de 2012. En cuanto al diálogo entre lectores, se pudo constatar que los que opinaron en la plataforma de Última Hora participaron activamente en un dialogo abierto y respetuoso, donde si bien no había muchas coincidencias de pensamientos e ideas, éstos no se agredían entre sí, como sí ocurrió en los espacios de la plataforma de $A B C$ Color, donde más que nada primó la imposición de pensamientos a través de agresiones ya sea a los actores involucrados en los artículos, como así también entre lectores.

\section{Referencias Bibliográficas}

Castells, M. (2009). Comunicación $y$ poder. Recuperado de https:// holismoplanetario.files.wordpress. com/2012/09/comunicacic3b3n_y_ poder_de_manuel_castells.pdf

Coordinadora por los Derechos Humanos del Paraguay (2012). Informe de derechos humanos sobre el Caso Marina Kue. Recuperado de http://www.codehupy.org/index. php?option=com_docman\&task=doc download\&gid $=18 \&$ Itemid $=21$

Moscovici, S. (1979). El psicoanálisis: su imagen y su público. Recuperado de http://taniars.files.wordpress. com / 2 oo 8/ o $2 / \mathrm{moscovici}$ - el psicoanalisis-su-imagen-y-su-publico. pdf

Rubio, J. (2009). Opinión pública y medios de comunicación. Teoría de la agenda setting. Revista Gazeta de Antropología, (25), 1-17. Recuperado de http://www. ugr.es/ pwlac/G25_o1JoseMaria_ Rubio_Ferreres.pdf

Cuevas, Y. (2011). Representaciones sociales en la prensa: aportaciones teóricas y metodológicas. Revista electrónica de Educación (SINÉCTICA), (36), 1-19. Recuperado de http://www. scielo.org. $\mathrm{mx} /$ scielo.php?pid=S1665109X2011000100003\&script=sci_arttext 